\title{
Resistance to Tan Spot and Stagonospora nodorum Blotch in Wheat-Alien Species Derivatives
}

\author{
R. E. Oliver and X. Cai, Department of Plant Sciences, North Dakota State University, Fargo 58105; R.-C. Wang, \\ United States Department of Agriculture-Agricultural Research Service (USDA-ARS), Forage and Range Research \\ Laboratory, Logan, UT 84322; and S. S. Xu and T. L. Friesen, USDA-ARS, Northern Crop Science Laboratory, \\ Fargo, ND 58105
}

\begin{abstract}
Oliver, R. E., Cai, X., Wang, R.-C., Xu, S. S., and Friesen, T. L. 2008. Resistance to tan spot and Stagonospora nodorum blotch in wheat-alien species derivatives. Plant Dis. 92:150-157.

Tan spot (caused by Pyrenophora tritici-repentis) and Stagonospora nodorum blotch (SNB) (caused by Stagonospora nodorum) are destructive fungal diseases of wheat (Triticum aestivum) throughout the world. Host plant resistance is thought to be an efficient and economical method of control. The objective of the present study was to identify novel sources of tan spot and SNB resistance in wheat genotypes derived from the crosses between wheat and alien species. Evaluations were conducted at the seedling stage in a growth chamber with $100 \%$ relative humidity. For each genotype, three replications were used for each disease. Among the 199 wheat-alien species derivatives evaluated, 65 exhibited resistance to tan spot and 30 showed resistance to SNB similar to BR34, a Brazilian wheat line used as the resistant control. Eleven derivatives were resistant to both diseases. Reactions of the derivatives and their respective wheat parents to tan spot and SNB suggest that resistance genes in the derivatives are derived from alien species. These derivatives can serve as desirable bridges for introgression of resistance genes from alien species to cultivated wheat, and could contribute novel and effective tan spot and SNB resistance to wheat breeding.
\end{abstract}

Tan spot is caused by the fungal pathogen Pyrenophora tritici-repentis (Died.) Drechsler, anamorph Drechslera triticirepentis (Died.) Shoemaker. Stagonospora nodorum blotch (SNB) is caused by Stagonospora nodorum (Berk.) E. Castell. \& Germano (syn. Septoria nodorum (Berk.) Berk.), teleomorph Phaeosphaeria nodorum (E. Müll.) Hedjar. (syn. Leptosphaeria nodorum E. Müll.). Both tan spot and SNB are common in many wheat-growing areas throughout the world and can cause substantial losses in yield and quality of grain $(12,35)$. In recent years, tan spot and SNB have become an increasing problem due to soil conservation practices such as no-tillage $(34,40)$, which leaves plant residues on the soil surface, allowing overwintering of the pathogen and a consequent increase in available inoculum. Increased disease severity also could be due to changes in pathogen virulence (17) or use of susceptible wheat cultivars $(24,41)$.

Several studies have characterized the genetics of tan spot and SNB resistance.

Corresponding author: T. L. Friesen

E-mail: timothy.friesent@ars.usda.gov

Accepted for publication 15 September 2007.

doi:10.1094/PDIS-92-1-0150

This article is in the public domain and not copyrightable. It may be freely reprinted with customary crediting of the source. The American Phytopathological Society, 2008.
Both qualitatively and quantitatively inherited resistance to tan spot and SNB have been identified in wheat $(18,19,23,38,41)$. Resistance genes have been identified on multiple wheat chromosomes (41). Stagonospora nodorum and Pyrenophora triticirepentis both are known to produce multiple host-selective toxins $(8,17,25-27)$, with ToxA being produced by both fungi (17). In the tan spot system, at least three hostselective toxins (HSTs) have been identified, with the genes encoding the proteinaceous toxins Ptr ToxA and Ptr ToxB having been cloned $(6,29)$. These HSTs have been shown to be highly important in disease development $(8,13,14,22)$.

SNB also has been shown recently to be an HST system, in which the fungus utilizes multiple proteinaceous toxins to cause disease. Similar to the tan spot system, host sensitivity or susceptibility genes identified to date are all dominant in nature, indicating an inverse gene-for-gene interaction between fungal-produced toxins and host susceptibility genes. Presently, HSTs have been identified, including SnToxA (17), SnTox1 (25), and SnTox2 (15). Each of these HST-host gene interactions has been shown to be highly important in disease development $(15,26,27)$. In addition to race-specific toxin sensitivity genes in the host, tan spot and SNB resistance quantitative trait loci (QTL) also have been identified $(7,10,41)$. For both SNB and tan spot, these QTL have been identified on several of the hexaploid wheat chromosomes, including $1 \mathrm{~B}, 3 \mathrm{~B}$, $3 \mathrm{D}$, and $5 \mathrm{~B}$ for tan spot and $1 \mathrm{~B}, 4 \mathrm{~B}, 6 \mathrm{~A}$, and $7 \mathrm{~B}$ for SNB $(7,27,41)$.

Although fungicide applications and cultural control practices can reduce disease severity and its detrimental effects $(9,37)$, host resistance is thought to provide the most economically efficient and sustainable method of control for both tan spot and SNB $(7,40)$. Most wheat cultivars, however, are susceptible to these diseases $(24,41)$ and, although sources of resistance have been identified, cultivars with complete resistance are not currently available $(1,33)$. Identification of novel sources of resistance to tan spot and SNB could aid in developing wheat cultivars resistant to these diseases.

Cultivated wheat generally has a narrow genetic base, which minimizes opportunities for developing host resistance to diseases; however, relatives of wheat contain valuable genes for wheat improvement $(11,20,21)$. Genes from alien species have been utilized to enhance genetic resistance to a number of fungal leaf diseases $(3,4,21,30)$. For example, more than 20 genes for resistance to leaf rust (caused by Puccinia triticina Erikss.) have been identified from wheat relatives (31). Characterization and introgression of selected genes has allowed reduction of linkage drag and deployment in wheat cultivars, facilitating host resistance to this disease (36). In the past 50 years, many wheat relatives have been hybridized with wheat, resulting in the production of numerous amphiploids, synthetic hexaploid wheat lines, and chromosome addition, substitution, and translocation lines. These genotypes offer excellent resources for identification and utilization of novel resistance genes, because they potentially combine alien resistance genes with a cultivated wheat background $(11,20)$. The objective of the present study was to identify novel sources of resistance to tan spot and SNB in these wheat genotypes. Utilization of these resistance sources in wheat breeding could enhance genetic diversity and aid in developing wheat cultivars with durable resistance to these diseases.

\section{MATERIALS AND METHODS}

Plant materials. In all, 199 wheat-alien species derivatives were evaluated for resistance to tan spot and SNB. These de- 
rivatives resulted from crosses between various wheat genotypes and 10 species of wheat relatives (Table 1). These wheatalien species derivatives were provided mainly by Dr. Stephen Jones, Department of Crop and Soil Sciences, Washington State University, Pullman; Dr. Peidu Chen, Department of Agronomy, Nanjing Agricultural University, China; and Dr. Yue Jin, United States Department of AgricultureAgricultural Research Service, University of Minnesota, St. Paul. Pedigrees, plant identification (PI) numbers, accession numbers, and chromosome constitutions of these derivatives, if known, are provided in Table 2. A Brazilian wheat line, BR34, and a North Dakota hard red spring wheat cultivar, Grandin, were used as resistant and susceptible controls, respectively, for evaluation of reaction to tan spot and SNB.

Evaluation for tan spot resistance. Evaluation for tan spot resistance followed the methods of $\mathrm{Xu}$ et al. (42) using the $P$. tritici-repentis isolate Pti2. Isolate Pti2 is a South Dakota race 1 field isolate which has been used in several previous studies $(10,16,42)$. Briefly, plants were grown in SC10 164-ml cone-containers (Stuewe and Sons, Inc. Corvallis OR), with three plants per cone and each genotype represented by three cones in each of three replicates, with each replicate being separated by time. Cones were arranged in racks of 98 , bordered by the susceptible control. Plants at the two- to three-leaf stage were sprayed with the spore suspension of isolate Pti2 of $P$. tritici-repentis race 1 at a concentration of 3,000 conidia $\mathrm{ml}^{-1}$ until run-off. Following inoculation, plants were kept in a growth chamber at $100 \%$ relative humidity at $21^{\circ} \mathrm{C}$ for $24 \mathrm{~h}$ with a $12-\mathrm{h}$ photoperiod, then transferred to a growth chamber at $21^{\circ} \mathrm{C}$ with a 12 -h photoperiod.

Disease evaluations were conducted 7 days after inoculation. Disease lesions were scored on a 1-to-5 scale, according to the rating system developed by Lamari and Bernier (22), where 1 is resistant (small dark-brown to black spots without surrounding chlorosis or tan necrosis), 2 is moderately resistant (small dark-brown to black spots with very little chlorosis or tan necrosis), 3 is moderately susceptible (small dark-brown to black spots com- pletely surrounded by a distinct chlorotic or tan necrotic ring, with lesions generally not coalescent), 4 is susceptible (small dark-brown or black spots completely surrounded with chlorotic or tan necrotic zones, with some lesions coalescent), and 5 is highly susceptible (dark-brown or black centers that may or may not be distinguishable, and most lesions consisting of coalescing chlorotic or tan necrotic zones). Genotypes with an equal amount of two lesion types were assigned an intermediate score.

Evaluation for SNB resistance. Evaluation for seedling SNB resistance was conducted using the same conditions and experimental design as described for $\tan$ spot. Inoculation with $S$. nodorum followed the methods of Liu et al. (27) and was performed at the two- to three-leaf stage, using isolate $\mathrm{Sn} 2000$. Sn2000 is a North Dakota field isolate used previously in disease evaluation, which is known to produce SnToxA and SnTox1 (17,2527,42). The spore suspension was adjusted to a concentration of $1 \times 10^{6}$ conidia ml ${ }^{-1}$, and disease evaluation was conducted 7 days after inoculation. Disease reaction was scored on a 0 -to-5 scale according to Liu et al. (27), where 0 is highly resistant (absence of visible lesions), 1 is resistant (few penetration points, with lesions consisting of flecking or small dark spots), 2 is moderately resistant (lesions consisting of dark spots with little surrounding necrosis or chlorosis), 3 is moderately susceptible (dark lesions completely, 2 to $3 \mathrm{~mm}$, surrounded by necrosis or chlorosis), 4 is susceptible (larger or chlorotic lesions, 4 $\mathrm{mm}$ or greater, with little coalescence), and 5 is highly susceptible (large coalescent lesions with very little green tissue remaining). Genotypes with equal numbers of two lesion types were assigned an intermediate score.

Statistical analysis. Analysis of variance (ANOVA) was conducted using the Statistical Analysis System (SAS, version 9.1; SAS Inc., Cary, NC) with Fisher's protected least significant difference used for mean separation between genotypes. To evaluate the effect of alien chromatin on a wheat background, derivatives with a common wheat parent were grouped and each pedigree group was analyzed sepa- rately. Susceptible and resistant controls were included in each ANOVA.

\section{RESULTS}

A large proportion of derivatives exhibited moderate to high levels of resistance to tan spot, SNB, or both. Of the 199 derivatives, more than half exhibited at least moderate SNB resistance and 150 had an SNB reaction significantly lower than Grandin, the susceptible control (Table 2). Among the 132 derivatives grouped according to a single wheat parent, 46 showed a significantly lower reaction than the respective wheat parental lines and 30 derivatives exhibited SNB reactions statistically similar to BR34, the resistant control. For tan spot, 111 derivatives had disease scores lower than Grandin and 65 derivatives showed resistance similar to BR34. In all, 44 derivatives showed a tan spot reaction significantly lower than their respective wheat parents and 37 derivatives were classified as resistant or moderately resistant. Of these 37 derivatives, however, 21 were also at least moderately resistant to SNB. A number of these derivatives have been shown to also contain genes for Fusarium head blight resistance (32). Thus, these genotypes represent a source of resistance to multiple diseases.

Of the 65 derivatives with tan spot resistance similar to BR34, 24 had the wheat cv. Chinese Spring as a parent. Chinese Spring is moderately susceptible to tan spot (Table 2). Within this set of derivatives, three had significantly higher resistance than Chinese Spring, including two disomic addition lines containing chromosomes $\mathrm{K}$ and $\mathrm{L}$ from Leymous racemosus (Table 2, no. 6 and 7), and a disomic substitution line containing chromosome 1E from Thinopyrum ponticum (Table 2, no. 22). Therefore, these alien chromosomes are likely to harbor genes for tan spot resistance. Interestingly, one line within this set of derivatives (Table 2, no. 31) was significantly more susceptible to tan spot than Chinese Spring. Of the 30 derivatives with SNB reactions similar to BR34, none were in the Chinese Spring group, although five derivatives (Table 2, no. 26, $28,31,32$, and 35) were more resistant to SNB than Chinese Spring.

Table 1. Wheat relatives used in the development of wheat-alien species derivatives

\begin{tabular}{lll}
\hline Species & Chromosome number & Genomes \\
\hline Aegilops tauschii (Coss.) Schmal. & $2 \mathrm{n}=2 \mathrm{x}=14$ & DD \\
Leymus racemosus Lam. & $2 \mathrm{n}=4 \mathrm{x}=28$ & NsNsXmXm \\
Elymus rectisetus (Nees) Á. Löve \& Connor & $2 \mathrm{n}=6 \mathrm{x}=42$ & StStYYWW \\
Thinopyrum junceum (L.) Love & $2 \mathrm{n}=6 \mathrm{x}=42$ & EEEEEE or JJJEE \\
Th. ponticum (Podp.) Z-W. Liu \& R.-C. Wang [syn. Agropyron elongatum (Host) Beauv., & \\
$\begin{array}{l}\text { Elytrigia pontica (Podp.) Holub., Lophopyrum ponticum (Podp.) Á. Löve] } \\
\text { Th. intermedium (Host) Barkworth \& D.R. Dewey }\end{array}$ & $2 \mathrm{n}=10 \mathrm{x}=70$ & EEEEEEEEEE \\
Th. elongatum (Host) D.R. Dewey & $2 \mathrm{n}=6 \mathrm{x}=42$ & EEEEStSt or JJEEStSt \\
Dasypyrum villosa L. & $2 \mathrm{n}=2 \mathrm{x}=14$ & EE \\
Avena sativa L. & $2 \mathrm{n}=2 \mathrm{x}=14$ & VV \\
Secale cereale L. & $2 \mathrm{n}=6 \mathrm{x}=42$ & AACCDD (differing from Triticeae) \\
\hline
\end{tabular}


Table 2. Mean seedling reactions of wheat-alien species derivatives to Stagonospora nodorum and Pyrenophora tritici-repentis ${ }^{\mathrm{a}}$

\begin{tabular}{|c|c|c|c|c|c|}
\hline \multirow[b]{2}{*}{ Code $^{b}$} & \multirow[b]{2}{*}{ Pedigree } & \multicolumn{2}{|c|}{ S. nodorum } & \multicolumn{2}{|c|}{ P. tritici-repentis } \\
\hline & & Score $^{c}$ & Reaction & Score $^{d}$ & Reaction \\
\hline BR34 & Triticum aestivum cultivar & 0.16 & HR & 1.35 & $\mathrm{R}$ \\
\hline Grandin & T. aestivum cultivar & 3.62 & MS & 3.88 & MS \\
\hline Chinese Spring (CS) & T. aestivum cultivar & 2.83 & MR & $2.67 *$ & MR \\
\hline 1 & CS-Leymous racemosus disomic addition line (DAL) $21 "+1 "[\mathrm{~A}]$ & 3.67 & MS & $2.33^{*}$ & MR \\
\hline 2 & CS-L. racemosus DAL $21 "+1 "[\mathrm{~F}]$ & 3.33 & MS & $2.67 *$ & MR \\
\hline 3 & CS-L. racemosus DAL 21"+1"[H] & 3.50 & MS & 3.33 & MS \\
\hline 4 & CS-L. racemosus DAL $21 "+1 "[\mathrm{I}]$ & 3.00 & MS & $2.50^{*}$ & MR \\
\hline 5 & CS-L. racemosus DAL $21 "+1 "[\mathrm{~J}]$ & 3.67 & MS & $2.33^{*}$ & MR \\
\hline 6 & CS-L. racemosus DAL $21 "+1 "[\mathrm{~K}]$ & $2.50 *$ & MR & $1.50 *$ & $\mathrm{R}$ \\
\hline 7 & CS-L. racemosus DAL $21 "+1 "[\mathrm{~L}]$ & 2.67 & MR & $1.17 *$ & $\mathrm{R}$ \\
\hline 8 & CS-L. racemosus DAL $21 "+1 "[\mathrm{~N}]$ & $2.00^{*}$ & MR & 3.67 & MS \\
\hline 9 & CS/Thinopyrum junceum DAL AJDAj1 & $2.50 *$ & MR & 2.83 & MR \\
\hline 10 & CS/Th. junceum DAL AJDAj2 & $2.50^{*}$ & MR & $1.67 *$ & $\mathrm{R}$ \\
\hline 11 & CS/Th. junceum DAL AJDAj3 & 2.67 & MR & $2.00^{*}$ & MR \\
\hline 12 & CS/Th. junceum DAL AJDAj4 & $2.33^{*}$ & MR & $1.83^{*}$ & $\mathrm{R}$ \\
\hline 13 & CS/Th. junceum DAL AJDAj5 & 3.17 & MS & $2.33^{*}$ & MR \\
\hline 14 & CS/Th. junceum DAL AJDAj6 & 3.00 & MS & $1.83^{*}$ & $\mathrm{R}$ \\
\hline 15 & CS/Th. junceum DAL AJDAj7 & 3.00 & MS & $2.00^{*}$ & MR \\
\hline 16 & CS/Th. junceum DAL AJDAj8 & 3.17 & MS & $2.50 *$ & MR \\
\hline 17 & CS/Th. junceum DAL AJDAj9 & 3.00 & MS & $2.17^{*}$ & MR \\
\hline 18 & CS/Th. junceum DAL AJDAj11 & 3.17 & MS & $2.00^{*}$ & MR \\
\hline 19 & CS/Th. junceum DAL HD3505 & 3.83 & MS & 3.00 & MS \\
\hline 20 & CS/Th. junceum DAL HD3508 & 5.00 & HS & $2.00 *$ & MR \\
\hline 21 & CS/Th. junceum DAL HD3515 & 3.50 & MS & $1.83^{*}$ & $\mathrm{R}$ \\
\hline 22 & Disomic CS-Th. ponticum substitution 1E/1D (2n = 42) & $2.17 *$ & MR & $1.50^{*}$ & $\mathrm{R}$ \\
\hline 23 & Disomic CS-Th. ponticum substitution 7E/7A $(2 n=42)$ & 2.67 & MR & $2.17 *$ & MR \\
\hline 24 & Disomic CS-Th. ponticum substitution 7E/7B $(2 \mathrm{n}=42)$ & $2.00^{*}$ & MR & $1.83^{*}$ & $\mathrm{R}$ \\
\hline 25 & Disomic CS-Th. ponticum substitution 7E/7D $(2 \mathrm{n}=42)$ & $2.17 *$ & MR & $2.17 *$ & MR \\
\hline 26 & CS/Th. ponticum $(\mathrm{PI} 206624) / / \mathrm{CS}$ & $1.83^{*}$ & $\mathrm{R}$ & $2.17 *$ & MR \\
\hline 27 & CS/Th. ponticum $(\mathrm{PI} 206624) / / \mathrm{CS}$ & 2.67 & MR & $2.33^{*}$ & MR \\
\hline 28 & CS/Th. ponticum (PI206624)//CS & $1.33^{*}$ & $\mathrm{R}$ & $2.67 *$ & MR \\
\hline 29 & CS/Th. intermedium (PI264770)//CS & $2.50^{*}$ & MR & $2.17 *$ & MR \\
\hline 30 & CS/Th. intermedium (PI264770)//CS & $2.50 *$ & MR & 2.83 & MR \\
\hline 31 & CS/Th. intermedium (PI264770)//CS & $1.50^{*}$ & $\mathrm{R}$ & 4.33 & $\mathrm{~S}$ \\
\hline 32 & CS/Th. intermedium (PI264770)//CS & $1.67 *$ & $\mathrm{R}$ & 3.33 & MS \\
\hline 33 & CS/Th. intermedium (PI264770)//CS & $2.33^{*}$ & MR & $2.50 *$ & MR \\
\hline 34 & CS/Th. intermedium (PI264770)//CS & 3.00 & MS & $2.17 *$ & MR \\
\hline 35 & CS ph1 mutant/Th. intermedium (PI264770)//CS & $1.83^{*}$ & $\mathrm{R}$ & $1.83^{*}$ & $\mathrm{R}$ \\
\hline 36 & CS ph1 mutant/Th. intermedium (PI264770)//CS & $2.33^{*}$ & MR & $2.17 *$ & MR \\
\hline LSD & $\ldots$ & 0.98 & $\ldots$ & 1.11 & $\ldots$ \\
\hline Madsen & Triticum aestivum cultivar & $2.17^{*}$ & MR & 3.83 & MS \\
\hline 37 & Madsen/Spitzer (wheat-Thinopyrum ponticum amphiploid) & $1.67 *$ & $\mathrm{R}$ & $2.67 *$ & MR \\
\hline 38 & Madsen/Spitzer & $2.33^{*}$ & MR & $2.83^{*}$ & MR \\
\hline 39 & Madsen/Spitzer & $1.50^{*}$ & $\mathrm{R}$ & $2.00^{*}$ & MR \\
\hline 40 & Madsen/Spitzer & $2.00 *$ & MR & 3.17 & MS \\
\hline 41 & Madsen/Spitzer & $1.50^{*}$ & $\mathrm{R}$ & 3.67 & MS \\
\hline 42 & Madsen/Spitzer & $1.67 *$ & $\mathrm{R}$ & $2.17 *$ & MR \\
\hline 43 & Madsen/Spitzer & $1.50^{*}$ & $\mathrm{R}$ & $2.83^{*}$ & MR \\
\hline 44 & Madsen/Spitzer & $1.67 *$ & $\mathrm{R}$ & 3.00 & MS \\
\hline 45 & Madsen/Spitzer & $2.50 *$ & MR & 3.33 & MS \\
\hline 46 & Madsen/Spitzer & $0.50^{*}$ & HR & 3.33 & MS \\
\hline 47 & Madsen/Spitzer & $1.17^{*}$ & $\mathrm{R}$ & 4.33 & $\mathrm{~S}$ \\
\hline 48 & Madsen/Spitzer & $2.83^{*}$ & MR & 3.83 & MS \\
\hline 49 & Madsen/Spitzer & $1.83^{*}$ & $\mathrm{R}$ & 3.50 & MS \\
\hline 50 & Madsen/Spitzer & $1.33^{*}$ & $\mathrm{R}$ & $2.83^{*}$ & MR \\
\hline 51 & Madsen/Spitzer & $2.33^{*}$ & MR & 3.00 & MS \\
\hline 52 & Madsen/Spitzer & $1.50 *$ & $\mathrm{R}$ & $2.83^{*}$ & MR \\
\hline 53 & Madsen/Spitzer & $2.50 *$ & MR & $2.50 *$ & MR \\
\hline 54 & Madsen/Spitzer & $2.17 *$ & MR & 3.67 & MS \\
\hline 55 & Madsen/Spitzer & $1.83^{*}$ & $\mathrm{R}$ & 4.17 & S \\
\hline 56 & Madsen/Spitzer & $1.50 *$ & $\mathrm{R}$ & 3.00 & MS \\
\hline 57 & Madsen/Spitzer & 3.67 & MS & 3.83 & MS \\
\hline
\end{tabular}

${ }^{a} \mathrm{HR}=$ highly resistant, $\mathrm{R}=$ resistant, $\mathrm{MR}=$ moderately resistant, $\mathrm{S}=$ susceptible, $\mathrm{HS}=$ highly susceptible, and $*$ significantly different from susceptible control (Grandin) at $P=0.05$.

${ }^{\mathrm{b}}$ BR34 $=$ resistant control, Grandin $=$ susceptible control, LSD $=$ least significant difference, and TCWL $178704=$ Turkish common wheat line 178704.

${ }^{\mathrm{c}}$ Stagonospora nodorum blotch evaluation: $0=$ highly resistant, $1=$ resistant, $2=$ moderately resistant, $3=$ moderately susceptible, $4=$ susceptible, and $5=$ highly susceptible.

${ }^{\mathrm{d}}$ Tan spot evaluation: 1 = resistant, 2 = moderately resistant, $3=$ moderately susceptible, $4=$ susceptible, and $5=$ highly susceptible.

e Thinopyrum turgidum cultivar.

${ }^{\mathrm{f}}$ Triticum aestivum cultivar.

$\mathrm{g}$ Wheat-Thinopyrum intermedium derivative. 
Table 2. (continued from preceding page)

\begin{tabular}{|c|c|c|c|c|c|}
\hline \multirow[b]{2}{*}{ Code $^{b}$} & \multirow[b]{2}{*}{ Pedigree } & \multicolumn{2}{|c|}{ S. nodorum } & \multicolumn{2}{|c|}{ P. tritici-repentis } \\
\hline & & Score $^{c}$ & Reaction & Score $^{d}$ & Reaction \\
\hline 58 & Madsen/Spitzer & 4.17 & $\mathrm{~S}$ & 3.17 & MS \\
\hline 59 & Madsen/Spitzer & 3.33 & MS & 3.17 & MS \\
\hline 60 & Madsen/Spitzer & $0.33 *$ & HR & 4.00 & $\mathrm{~S}$ \\
\hline 61 & Madsen/Spitzer & $0.33^{*}$ & $\mathrm{HR}$ & $2.50 *$ & MR \\
\hline 62 & Madsen/Spitzer & $1.50 *$ & $\mathrm{R}$ & 3.33 & MS \\
\hline 63 & Madsen/Spitzer & $0.33^{*}$ & HR & $2.83^{*}$ & MR \\
\hline 64 & Madsen/Spitzer & $0.17^{*}$ & $\mathrm{HR}$ & $1.50 *$ & $\mathrm{R}$ \\
\hline 65 & Spitzer/Madsen & $2.33^{*}$ & MR & $2.67 *$ & MR \\
\hline 66 & Spitzer/Madsen & $0.33^{*}$ & HR & 3.67 & MS \\
\hline 67 & Spitzer/Madsen & $1.83^{*}$ & $\mathrm{R}$ & $2.83^{*}$ & MR \\
\hline 68 & Spitzer/Madsen & $1.50 *$ & $\mathrm{R}$ & 3.33 & MS \\
\hline 69 & Spitzer/Madsen & $1.67 *$ & $\mathrm{R}$ & 3.17 & MS \\
\hline 70 & Spitzer/Madsen & $1.83^{*}$ & $\mathrm{R}$ & 4.17 & $\mathrm{~S}$ \\
\hline 71 & Spitzer/Madsen & $1.67 *$ & $\mathrm{R}$ & 3.50 & MS \\
\hline 72 & Spitzer/Madsen & $2.50 *$ & MR & 3.67 & MS \\
\hline 73 & Spitzer/Madsen & $2.17 *$ & MR & 4.00 & $\mathrm{~S}$ \\
\hline 74 & Spitzer/Madsen & $1.00 *$ & $\mathrm{R}$ & 3.00 & MS \\
\hline 75 & Spitzer/Madsen & $1.17 *$ & $\mathrm{R}$ & 3.33 & MS \\
\hline 76 & Spitzer/Madsen & $1.50 *$ & $\mathrm{R}$ & $2.83^{*}$ & MR \\
\hline 77 & Spitzer/Madsen & $2.33^{*}$ & MR & 4.67 & $\mathrm{~S}$ \\
\hline 78 & Spitzer/Madsen & $0.33^{*}$ & HR & 4.00 & $\mathrm{~S}$ \\
\hline 79 & Spitzer/Madsen & $2.00 *$ & MR & $2.83^{*}$ & MR \\
\hline 80 & Spitzer/Madsen & $0.17 *$ & $\mathrm{HR}$ & 3.50 & MS \\
\hline 81 & Spitzer/Madsen & $0.83^{*}$ & $\mathrm{R}$ & 4.33 & $\mathrm{~S}$ \\
\hline 82 & Spitzer/Madsen & $0.83^{*}$ & $\mathrm{R}$ & 3.83 & MS \\
\hline 83 & Spitzer/Madsen & $2.00 *$ & MR & 3.33 & MS \\
\hline 84 & Spitzer/Madsen & $1.67 *$ & $\mathrm{R}$ & 3.67 & MS \\
\hline 85 & Spitzer/Madsen & $1.83^{*}$ & $\mathrm{R}$ & 3.83 & MS \\
\hline 86 & Spitzer/Madsen & $0.33^{*}$ & HR & $2.50 *$ & MR \\
\hline 87 & Spitzer/Madsen & $1.33^{*}$ & $\mathrm{R}$ & 4.00 & $\mathrm{~S}$ \\
\hline 88 & Spitzer/Madsen & $1.33^{*}$ & $\mathrm{R}$ & 3.83 & MS \\
\hline 89 & Spitzer/Madsen & $1.50 *$ & $\mathrm{R}$ & 3.83 & MS \\
\hline 90 & Spitzer/Madsen & $1.17 *$ & $\mathrm{R}$ & $2.33^{*}$ & MR \\
\hline 91 & Spitzer/Madsen & $1.00 *$ & $\mathrm{R}$ & $2.00 *$ & MR \\
\hline 92 & Spitzer/Madsen & $1.17 *$ & $\mathrm{R}$ & $2.67 *$ & MR \\
\hline 93 & Spitzer/Madsen & $1.00 *$ & $\mathrm{R}$ & $2.50 *$ & MR \\
\hline 94 & Spitzer/Madsen & $1.83^{*}$ & $\mathrm{R}$ & 3.83 & MS \\
\hline 95 & Spitzer/Madsen & $1.17 *$ & $\mathrm{R}$ & $2.67 *$ & MR \\
\hline 96 & Spitzer/Madsen & $0.50 *$ & HR & 3.00 & MS \\
\hline 97 & Spitzer/Madsen & $2.00 *$ & MR & $2.33^{*}$ & MR \\
\hline 98 & Spitzer/Madsen & $2.00 *$ & MR & 4.17 & $\mathrm{~S}$ \\
\hline 99 & Spitzer/Madsen & $1.67 *$ & $\mathrm{R}$ & 4.33 & $\mathrm{~S}$ \\
\hline 100 & Spitzer/Madsen & $2.17 *$ & MR & 4.17 & $\mathrm{~S}$ \\
\hline 101 & Spitzer/Madsen & $0.67 *$ & $\mathrm{R}$ & $2.50 *$ & MR \\
\hline 102 & Spitzer/Madsen & $1.00 *$ & $\mathrm{R}$ & $2.50 *$ & $\mathrm{MR}$ \\
\hline LSD & $\ldots$ & 0.69 & $\ldots$ & 1.04 & $\ldots$ \\
\hline TCWL 178704 & Triticum aestivum cultivar & $1.33 *$ & $\mathrm{R}$ & 3.33 & MS \\
\hline 103 & TCWL 178704-Thinopyrum ponticum derivative & $2.00 *$ & $\mathrm{MR}$ & 4.00 & $\mathrm{~S}$ \\
\hline 104 & TCWL 178704-Th. ponticum derivative & $0.83 *$ & $\mathrm{R}$ & $1.67 *$ & $\mathrm{R}$ \\
\hline 105 & TCWL 178704-Th. ponticum derivative & $1.83^{*}$ & $\mathrm{R}$ & $2.83^{*}$ & MR \\
\hline 106 & TCWL 178704-Th. ponticum derivative $(2 \mathrm{n}=46)$ & $0.17 *$ & HR & $1.83^{*}$ & $\mathrm{R}$ \\
\hline 107 & TCWL 178704-Th. ponticum derivative $(2 \mathrm{n}=46)$ & $0.67 *$ & $\mathrm{R}$ & $1.83^{*}$ & $\mathrm{R}$ \\
\hline 108 & TCWL $178704-T h$. ponticum derivative $(2 \mathrm{n}=47)$ & $0.33 *$ & $\mathrm{HR}$ & $1.67 *$ & $\mathrm{R}$ \\
\hline 109 & TCWL 178704-Th. ponticum derivative $(2 \mathrm{n}=47)$ & $0.00 *$ & HR & $1.67 *$ & $\mathrm{R}$ \\
\hline 110 & TCWL $178704-T h$. ponticum derivative $(2 n=47)$ & $0.17 *$ & $\mathrm{HR}$ & $1.83^{*}$ & $\mathrm{R}$ \\
\hline 111 & TCWL 178704-Th. ponticum derivative $(2 \mathrm{n}=47)$ & $0.50 *$ & HR & $2.17 *$ & MR \\
\hline 112 & TCWL $178704-T h$. ponticum derivative $(2 \mathrm{n}=47)$ & $0.33 *$ & $\mathrm{HR}$ & $1.83 *$ & $\mathrm{R}$ \\
\hline 113 & TCWL $178704-T h$. ponticum derivative $(2 n=47)$ & $1.67 *$ & $\mathrm{R}$ & $2.67 *$ & MR \\
\hline 114 & TCWL 178704-Th. ponticum derivative $(2 \mathrm{n}=47)$ & $0.67 *$ & $\mathrm{R}$ & $1.50 *$ & $\mathrm{R}$ \\
\hline 115 & TCWL $178704-T h$. ponticum derivative $(2 \mathrm{n}=48)$ & $0.17 *$ & $\mathrm{HR}$ & $1.83^{*}$ & $\mathrm{R}$ \\
\hline 116 & TCWL 178704-Th. ponticum derivative $(2 \mathrm{n}=48)$ & $1.17 *$ & $\mathrm{R}$ & $1.83^{*}$ & $\mathrm{R}$ \\
\hline 117 & TCWL $178704-T h$. ponticum derivative $(2 \mathrm{n}=48)$ & $0.50 *$ & HR & $2.17 *$ & MR \\
\hline 118 & TCWL $178704-T h$. ponticum derivative $(2 n=57)$ & $0.50 *$ & $\mathrm{HR}$ & $2.00 *$ & $\mathrm{MR}$ \\
\hline 119 & TCWL $178704-T h$. ponticum derivative $(2 \mathrm{n}=68)$ & $1.17 *$ & MR & $2.50 *$ & MR \\
\hline 120 & TCWL $178704-T h$. ponticum derivative $(2 \mathrm{n}=70)$ & $1.33 *$ & $\mathrm{R}$ & $2.00 *$ & MR \\
\hline 121 & TCWL $178704-T h$. ponticum derivative $(2 \mathrm{n}=70)$ & $2.50 *$ & MR & $1.67 *$ & $\mathrm{R}$ \\
\hline 122 & TCWL $178704-T h$. ponticum derivative $(2 \mathrm{n}=70)$ & $3.00 *$ & MS & $2.67 *$ & MR \\
\hline LSD & $\ldots$ & 0.47 & $\ldots$ & 0.98 & $\ldots$ \\
\hline Langdon & Triticum turgidum cultivar & 3.50 & MS & 4.17 & $\ddot{S}$ \\
\hline 123 & Langdon/Aegilops tauschii (RL5560) & 3.67 & MS & $1.83^{*}$ & $\mathrm{R}$ \\
\hline \multirow[t]{2}{*}{124} & Langdon/Ae. tauschii (PI268210) & $0.67 *$ & $\mathrm{R}$ & $2.17 *$ & MR \\
\hline & & & & \multicolumn{2}{|c|}{ (continued on next page) } \\
\hline
\end{tabular}


Table 2. (continued from preceding page)

\begin{tabular}{|c|c|c|c|c|c|}
\hline \multirow[b]{2}{*}{ Code $^{b}$} & \multirow[b]{2}{*}{ Pedigree } & \multicolumn{2}{|c|}{ S. nodorum } & \multicolumn{2}{|c|}{ P. tritici-repentis } \\
\hline & & Score $^{c}$ & Reaction & Score $^{d}$ & Reaction \\
\hline 125 & KAD1 (Langdon-Thinopyrum ponticum amphiploid, $2 \mathrm{n}=42$ ) & $2.83 *$ & MR & $2.67 *$ & MR \\
\hline 126 & KAD7 (Langdon-Th. ponticum amphiploid, $2 \mathrm{n}=42$ ) & $2.33^{*}$ & MR & $2.83^{*}$ & MR \\
\hline 127 & KAD8 (Langdon-Th. ponticum amphiploid, $2 \mathrm{n}=42$ ) & $2.50 *$ & MR & $2.17 *$ & MR \\
\hline 128 & KAD9 (Langdon-Th. ponticum amphiploid, $2 \mathrm{n}=42$ ) & $2.17 *$ & MR & $2.83^{*}$ & MR \\
\hline LSD & $\ldots$ & 0.50 & $\ldots$ & 0.96 & $\ldots$ \\
\hline Fukuhokomuji & Triticum aestivum cultivar & 4.17 & $\mathrm{~S}$ & 3.83 & MS \\
\hline 129 & Fukuhokomugi/Elymus rectisetus DAL A1026 & 4.17 & $\mathrm{~S}$ & $3.17 *$ & MS \\
\hline 130 & Fukuhokomugi/E. rectisetus DAL A1034 & 4.17 & $\mathrm{~S}$ & $3.17 *$ & MS \\
\hline 131 & Fukuhokomugi/E. rectisetus DAL A1048 & 4.50 & $\mathrm{~S}$ & $3.17 *$ & MS \\
\hline 132 & Fukuhokomugi/E. rectisetus DAL A1057 & $2.17 *$ & MR & $2.50^{*}$ & MR \\
\hline LSD & $\ldots$ & 0.52 & $\ldots$ & 0.62 & $\ldots$ \\
\hline \multicolumn{6}{|l|}{ Other } \\
\hline 133 & Botno $\mathrm{e} /$ Ae tauschii $(625)$ & 3.17 & MS & $2.33^{*}$ & MR \\
\hline 134 & CS/Thinopyrum ponticum (PI206624)//Madsen & $2.17 *$ & MR & $2.17 *$ & MR \\
\hline 135 & CS/Th. ponticum (PI206624)//Madsen & $2.50 *$ & MR & $1.67^{*}$ & $\mathrm{R}$ \\
\hline 136 & CS/Th. ponticum (PI206624)//CS///Madsen & $2.17 *$ & MR & 3.17 & MS \\
\hline 137 & CS/Th. ponticum (PI206624)//CS///Madsen & $2.00 *$ & MR & $2.50 *$ & MR \\
\hline 138 & CS/Th. ponticum (PI206624)//CS///Madsen & $2.00 *$ & MR & 4.17 & $\mathrm{~S}$ \\
\hline 139 & CS ph1 mutant/Th. ponticum (PI206624)//Madsen & $1.33^{*}$ & $\mathrm{R}$ & $2.33^{*}$ & MR \\
\hline 140 & CS/Th. intermedium (PI264770)//Madsen & $1.33 *$ & $\mathrm{R}$ & 3.83 & MS \\
\hline 141 & CS/Th. intermedium (PI264770)//CS///Madsen & $2.33^{*}$ & MR & 3.50 & MS \\
\hline 142 & CS/Th. intermedium (PI264770)//CS///Madsen & $1.50 *$ & $\mathrm{R}$ & 4.00 & $\mathrm{~S}$ \\
\hline 143 & CS/Th. intermedium (PI264770)//CS///Madsen & 3.00 & MS & $2.33^{*}$ & MR \\
\hline 144 & CS/Th. intermedium (PI264770)//CS///Madsen & $1.50 *$ & $\mathrm{R}$ & 3.00 & MS \\
\hline 145 & CS/Th. intermedium (PI264770)//CS///Madsen & $2.33^{*}$ & MR & $2.67 *$ & MR \\
\hline 146 & CS/Th. intermedium (PI264770)//CS///Madsen & $2.00 *$ & MR & 3.33 & MS \\
\hline 147 & CS/Th. intermedium (PI264770)//CS///Madsen & $2.17 *$ & MR & $2.17^{*}$ & MR \\
\hline 148 & CS/Th. intermedium (PI264770)//CS///Madsen & $2.17 *$ & MR & 2.83 & MR \\
\hline 149 & CS/Th. intermedium (PI264770)//CS///Madsen & $2.00 *$ & MR & 3.50 & MS \\
\hline 150 & CS/Th. intermedium (PI264770)//CS///Madsen & $1.67 *$ & $\mathrm{R}$ & $2.67 *$ & MR \\
\hline 151 & CS/Th. intermedium (PI264770)//CS///Madsen & $2.67 *$ & MR & 3.67 & MS \\
\hline 152 & CS ph1 mutant/Th. intermedium (PI264770)//CS///Madsen & 3.17 & MS & 3.83 & MS \\
\hline 153 & CS ph1 mutant/Th. intermedium (PI264770)//CS///Madsen & $1.50 *$ & $\mathrm{R}$ & 3.00 & MS \\
\hline 154 & CS ph1 mutant/Th. intermedium (PI264770)//CS///Madsen & $1.50 *$ & $\mathrm{R}$ & $2.17 *$ & MR \\
\hline 155 & CS ph1 mutant/Th. intermedium (PI264770)//CS///Madsen & $2.50 *$ & MR & $2.50^{*}$ & MR \\
\hline 156 & TA3392 (Wheat-Th. intermedium amphiploid) & $0.33 *$ & HR & $2.50 *$ & MR \\
\hline 157 & TA3409 (Wheat-Agropyron distichum amphiploid) & 3.00 & MS & 3.33 & MS \\
\hline 158 & TA3425 (CS/Ag. elongatum) & $1.33^{*}$ & $\mathrm{R}$ & $1.50 *$ & $\mathrm{R}$ \\
\hline 159 & TA3426 (CS/Ag. scripeum amphiploid) & $2.33^{*}$ & MR & $1.83^{*}$ & $\mathrm{R}$ \\
\hline 160 & TA4014 (Agent 3DL Lr24) & 3.67 & MS & 3.33 & MS \\
\hline 161 & TA5033 (TAM101 Ti4AS·4AL-6RL-4AL H25) & $1.83 *$ & $\mathrm{R}$ & 3.33 & MS \\
\hline 162 & TA5041 (TAM104 T6B·6RL Pm20) & 2.83 & MR & $2.50 *$ & MR \\
\hline 163 & TA5501 (Heine IV T1DS·1DL-7Ai\#2L Lr38) & $0.67 *$ & $\mathrm{R}$ & 3.50 & MS \\
\hline 164 & TA5502 (Heine IV T7Ai\#2L-5AS·5AL Lr38) & $1.67 *$ & $\mathrm{R}$ & 3.67 & MS \\
\hline 165 & TA5503 (Heine IV T6DS-6DL-7Ai\#2L Lr38) & $1.33 *$ & $\mathrm{R}$ & 3.50 & MS \\
\hline 166 & TA5504 (Wheat-Ag. intermedium translocation T3DL·3DS-7Ai\#2L) & $1.83 *$ & $\mathrm{R}$ & $2.00 *$ & MR \\
\hline 167 & TA5505 (Heine IV T2AS·2AL-3DS·3DL $L r 38)$ & $0.67 *$ & $\mathrm{R}$ & 3.00 & MS \\
\hline 168 & TA8023 (wheat-Th. junceum amphiploid, AABBDDJJ) $(2 \mathrm{n}=56)$ & $1.50 *$ & $\mathrm{R}$ & $1.33^{*}$ & $\mathrm{R}$ \\
\hline 169 & AgCs (CS-Th. elongatum amphiploid, 2n = 56) & $1.50 *$ & $\mathrm{R}$ & $1.33^{*}$ & $\mathrm{R}$ \\
\hline 170 & PI550711 (wheat-Thinopyrum amphiploid) & 3.17 & MS & 3.50 & MS \\
\hline 171 & XC97-1-55 (wheat-Thinopyrum amphiploid) & $1.00 *$ & $\mathrm{R}$ & $2.00 *$ & MR \\
\hline 172 & Eltan $\mathrm{f} / T h$. ponticum (PI206624) & $1.67 *$ & $\mathrm{R}$ & $2.17^{*}$ & MR \\
\hline 173 & PI550715 (wheat-Th. ponticum amphiploid, $2 \mathrm{n}=56$ ) & $0.50 *$ & HR & 3.00 & MS \\
\hline 174 & PI550715/Eltan & $0.83^{*}$ & $\mathrm{R}$ & $2.17 *$ & MR \\
\hline 175 & PI550715/Spitzer (blue seeds) & $0.17 *$ & HR & $2.50 *$ & MR \\
\hline 176 & 6VS-6AL translocation/Yang $158^{\mathrm{f}}$ & 4.00 & $\mathrm{~S}$ & 3.00 & MS \\
\hline 177 & Yang158/6VS-6AL translocation//Yang158 & 4.00 & $\mathrm{~S}$ & 2.83 & MR \\
\hline 178 & Wheat-Dasypyrum villosa 6VS/6AL translocation & $2.67 *$ & MR & 3.00 & MS \\
\hline 179 & 6VS/6AL trans./Yangmai5 $5^{\mathrm{f} * 3 / / \text { Sheng } 32109^{\mathrm{f}}}$ & 3.83 & MS & 3.17 & MS \\
\hline 180 & (CS/3/Xiangmai1 1 /D. villosa//Xiangmai1)/(6VS-6AL translocation/Yang158) & 3.67 & MS & 3.00 & MS \\
\hline 181 & CS/L. racemosus//CS/3/Wangshuibai ${ }^{\mathrm{f}}$ & $0.67 *$ & $\mathrm{R}$ & $2.67 *$ & MR \\
\hline 182 & 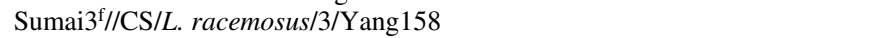 & $0.17 *$ & HR & $2.50 *$ & MR \\
\hline 183 & Sumai3//CS/L. racemosus/3/Yang158 & 3.00 & MS & 4.50 & $\mathrm{~S}$ \\
\hline 184 & CS/L. racemosus /3/Xiangmai1/ Haynaldia villosa//Xiangmai1 & $1.67 *$ & $\mathrm{R}$ & 3.83 & MS \\
\hline 185 & 3D-Agropyron transfer \#11 & $1.83^{*}$ & $\mathrm{R}$ & $2.67 *$ & MR \\
\hline 186 & 3D-Agropyron transfer \#16 & $1.67 *$ & $\mathrm{R}$ & 2.83 & MR \\
\hline 187 & GN775 (Triticum durum/Aegilops tauschii//Ae. ventricosa/3/oat) & $1.33 *$ & $\mathrm{R}$ & $2.33^{*}$ & MR \\
\hline 188 & Amigo (wheat-rye and Thinopyrum ponticum translocation) & $1.17 *$ & $\mathrm{R}$ & 3.00 & MS \\
\hline 189 & MT-2g/Stephens ${ }^{\mathrm{f}}$ & 3.17 & MS & 2.83 & MR \\
\hline 190 & MT-2/Stephens & $1.83 *$ & $\mathrm{R}$ & 4.50 & $\mathrm{~S}$ \\
\hline \multirow[t]{2}{*}{191} & MT-2/Stephens & 2.83 & MR & $2.50^{*}$ & MR \\
\hline & & & & \multicolumn{2}{|c|}{ (continued on next page) } \\
\hline
\end{tabular}


Table 2. (continued from preceding page)

\begin{tabular}{|c|c|c|c|c|c|}
\hline \multirow[b]{2}{*}{ Code $^{b}$} & \multirow[b]{2}{*}{ Pedigree } & \multicolumn{2}{|c|}{ S. nodorum } & \multicolumn{2}{|c|}{ P. tritici-repentis } \\
\hline & & Score $^{c}$ & Reaction & Score $^{d}$ & Reaction \\
\hline 192 & Stephens//Harlan JR35e/Maroccos $/ / /$ Stephens & $1.17 *$ & $\mathrm{R}$ & 2.83 & MR \\
\hline 193 & Yangmai5 & $1.33^{*}$ & $\mathrm{R}$ & $2.50 *$ & MR \\
\hline 194 & Yangmai158 & 3.83 & MS & 2.83 & MR \\
\hline 195 & Yangmai $10^{\mathrm{f}}$ & 3.83 & MS & 3.17 & MS \\
\hline 196 & Sumai 3 (purple anthers) & 3.33 & MS & 3.67 & MS \\
\hline 197 & C04-13 (CS/Ae. speltoides) & $0.67 *$ & $\mathrm{R}$ & $2.50 *$ & MR \\
\hline 198 & C08-4 (CS/Ae. speltoides) & 3.17 & MS & 2.83 & MR \\
\hline 199 & C11-5 (CS/Ae. speltoides) & 3.17 & MS & $2.33^{*}$ & MR \\
\hline LSD & $\ldots$ & 0.83 & $\mathrm{R}$ & 1.08 & $\mathrm{R}$ \\
\hline
\end{tabular}

Sixty-six lines were derived from the cross between 'Madsen' and 'Spitzer' (X. Cai and S. S. Jones, unpublished). Madsen, a common soft white winter wheat cultivar, had a lower SNB reaction than the susceptible check, Grandin. Spitzer, a partial wheat- $T$. ponticum amphiploid, was not tested in this study. Of the 66 MadsenSpitzer lines evaluated, 16 were more resistant to SNB than Madsen and 6 (Table 2, no. 39, 42, 64, 90, 91, and 97) exhibited tan spot reactions similar to BR34. For SNB, of 23 Madsen-Spitzer derivatives that were more resistant than Madsen, 13 (Table 2, no. 46, 60, 61, 63, 64, 66, 78, 80$82,86,96$, and 101) had reactions comparable to BR34. Derivative no. 64 exhibited excellent resistance to both SNB and tan spot, whereas lines 57, 58, and 59 showed significantly higher SNB disease reactions than the Madsen parental line. The disease reactions of Spitzer are unknown; however, the high level of resistance observed in several derivatives suggests that Spitzer, or gene interaction in the progeny lines, may contribute to this resistance. None of the lines in this subset of material had a significantly more susceptible tan spot disease reaction than Madsen.

The Turkish common wheat line 178704, a parent to 20 derivatives evaluated in this study, was moderately susceptible to tan spot (Table 2). Fifteen of the derivatives (Table 2 , no. 104, 106-112, $114-118,120$, and 121) were significantly more resistant than the Turkish wheat parent and exhibited tan spot reactions comparable to BR34. These derivatives contain various amounts of $T$. ponticum chromatin, again suggesting that this species is a valuable source of resistance to tan spot. No derivatives in this subset had a significantly higher tan spot disease reaction than the parental Turkish common wheat line. More than half of the Turkish common wheat line derivatives exhibited SNB resistance significantly greater than the wheat parent, including one derivative with apparent immunity (Table 2, no. 109). Nine derivatives had SNB resistance similar to BR34 (Table 2, no. 108-112, 115, 117, and 118). These nine derivatives also exhibited comparably high levels of resistance to tan spot. Although four of the Turkish com- mon wheat line derivatives had significantly higher SNB disease reactions, none were highly susceptible, with the highest average disease reaction among these lines being 3.0 on the 0 -to- 5 scale.

Langdon, a durum wheat cultivar, was susceptible to tan spot (Table 2). The six Langdon-alien species derivatives contained chromatin from either Aegilops tauschii or $T$. ponticum and were all significantly more resistant than Langdon. In addition, three lines (Table 2, no. 123, 124, and 127) were not significantly different from BR34, showing a high level of tan spot resistance. Five of the six Langdon derivatives exhibited an SNB reaction significantly lower than Langdon, with no. 124 having the highest level of resistance in this group.

Fukuhokomuji, a Japanese common wheat cultivar, has been used to develop disomic addition lines containing chromosomes from the alien species Elymus rectisetus. Although all four lines evaluated in this study were significantly more resistant than the wheat parent for tan spot, none were comparable to BR34 (Table 2). For both SNB and tan spot, one derivative (Table 2, no. 132) was significantly more resistant than the wheat parent.

Derivatives with complex pedigrees or unavailable wheat parents were grouped together. Of these 67 derivatives, 17 exhibited $\tan$ spot reactions similar to BR34, with no. 135, 158, 159, 168, and 169 having the highest levels of tan spot resistance in this group (Table 2). Nine derivatives had SNB resistance similar to BR34 (Table 2, no. 156, 163, 167, 173-175, 181, 182, and 197). These derivatives were highly resistant, with SNB reactions approaching immunity. Although these pedigrees complicate identification of the resistance sources, all highly resistant derivatives include a parental Thinopyrum sp. It seems likely, therefore, that resistance genes are carried by several species within this genus.

Disease reactions of the wheat parents ranged from susceptible to moderately resistant, with the majority exhibiting moderate susceptibility to both diseases. Therefore, it appears that the alien species in these derivatives harbor both tan spot and SNB resistance genes, and that these genes have been transferred to the wheatalien species derivatives.

\section{DISCUSSION}

We identified genotypes with resistance to tan spot and SNB from 199 wheat-alien species derivatives, in which $A$. tauschii, $L$. racemosus, E. rectisetus, $T$. ponticum, $T$. junceum, T. intermedium, T. elongatum, Dasypyrum villosa, Avena sativa, and Secale cereale were involved. Resistance in these derivatives could result from the alien species and, thus, be derived from genomes different from those in wheat. These derivatives, therefore, may represent novel sources of resistance to both diseases. Introgression and pyramiding of alien resistance genes and currently identified resistance genes in the primary gene pool will broaden the genetic variability of wheat and strengthen resistance of wheat to these diseases.

Ten different alien species were involved in the development of the derivatives evaluated in this study. A number of derivatives exhibited significantly higher levels of resistance to tan spot, SNB, or both than their respective wheat parents, suggesting that alien chromatin confers resistance in these derivatives. Alien chromatin present in these derivatives has not been characterized; therefore, little information can be extrapolated from the present data as to what alien chromosomes harbor resistance genes. Further cytogenetic and molecular characterization will be needed for these derivatives to be efficiently utilized in wheat breeding.

The genome differences, however, have been a major challenge for gene introgression from these alien species into wheat in terms of hybridization between wheat and the wild species and recombination between wheat and alien chromosomes. The resistant wheat-alien species derivatives identified in this study include amphiploids and alien chromosome addition, substitution, and translocation lines. Many of these derivatives contain large amounts of alien chromatin, which harbor genes conditioning undesirable traits such as late maturity and low yield potential in addition to resistance genes. Therefore, they 
may not be utilized directly in breeding for tan spot and SNB resistance. They are, however, ideal "bridge" materials for introgression of resistance genes into wheat genomes and development of elite breederfriendly germplasm with resistance to both diseases. On the other hand, some of the resistant alien chromosome translocation lines may contain small amounts of alien chromatin without obvious linkage drag. These lines could be used directly in cultivar development without prebreeding efforts.

Resistant derivatives with large amounts of alien chromatin may be engineered to eliminate unwanted alien chromatin and genes using the phlb mutant and homologous pairing promoters such as the $P h^{I}$ gene (5). This will enhance recombination between wheat and alien chromatin and lead to production of wheat-alien chromosome translocation lines. Gene transfer is further enhanced by molecular cytogenetic techniques such as fluorescent in situ hybridization (FISH), which reveals the amount and location of alien chromatin (39). Introgression of the identified resistance genes to a cultivated wheat background is underway. This prebreeding phase could minimize linkage drag and improve the agronomic characteristics and adaptation of the progeny, producing breeder-friendly, disease-resistant germplasm. Eventually, this research will allow wheat breeders access to the alien gene pool and facilitate development of broadspectrum resistance to these diseases.

Both the tan spot and SNB systems are known to involve host-selective toxins, which correspond to dominant host sensitivity genes in an inverse gene-for-gene manner $(15,28)$. Each of these toxin-host gene interactions have been shown to be highly important in disease development $(7,15,26,27)$. Several other resistance factors that have not been connected with HST production in the pathogen also have been identified. Faris and Friesen (10) identified several QTL that were effective across several races, including races producing Ptr ToxA, Ptr ToxB, and Ptr ToxC. This study showed that a high level of resistance can be obtained even in the presence of toxin sensitivity. This is also the case in the SNB system, where qualitative or quantitative resistance genes have been identified on at least 19 of the 21 hexaploid wheat chromosomes (41).

In addition to identifying a major toxin sensitivity gene on chromosome $1 \mathrm{~B}$, Liu et al. (27) identified several resistance QTL that were not associated with toxin sensitivity. The presence of non-toxinassociated resistance loci likely indicates various forms of active resistance that, if pyramided, could potentially lead to high levels of resistance. Friesen et al. (15) showed that some lines sensitive to SnToxA were still highly resistant to a SnToxA-producing Stagonospora nodorum isolate if other nontoxin-associated resistance QTL were present in the lines. This would also indicate, similar to the tan spot system, that nontoxin-associated resistance can be highly effective even in the presence of toxin sensitivity genes.

Collectively, these studies show the importance of identifying and utilizing different sources of resistance for effective control of both tan spot and SNB. In the present study, several of the derivatives acquired higher levels of resistance by gaining chromosomes from other species. This would indicate that nontoxinassociated forms of resistance are being added. This would be the assumption because all known toxin sensitivities in these two systems are known to be inherited in a dominant fashion. Therefore, if additional toxin sensitivity were added, resistance would be decreased rather than increased. However, an increase in susceptibility compared with the parent from which the line was derived was identified in several derivatives for SNB and one derivative for tan spot (line no. 31). For SNB, these lines with increased susceptibility (line no. 19, 20, 57, 58, 59, 103, 105, 121, and 122) occurred in the Chinese Spring, Madsen/ Spitzer, and Turkish common wheat groups. Due to the toxin-producing ability of $P$. tritici-repentis and $S$. nodorum, it is highly likely that alien chromatin in the derivatives contains toxin sensitivity genes not present in the wheat parental lines. It also is possible that suppressors of resistance could be present in the alien chromatin, which shut down an active host resistance mechanism (2). More analysis of these derivatives, including population development, is needed to identify the mechanism of increased susceptibility.

\section{ACKNOWLEDGMENTS}

We thank D. J. Holmes, A. R. Little, and P. T. Meyer for technical assistance; and $\mathrm{H}$. Tsujimoto of Tottori University, Japan, for providing seed.

\section{LITERATURE CITED}

1. Aguilar, V., Stamp, P., Winzeler, M., Winzeler, H., Schachermayr, G., Keller, B., Zanetti, S., and Messmer, M. M. 2005. Inheritance of field resistance to Stagonospora nodorum leaf and glume blotch and correlations with other morphological traits in hexaploid wheat (Triticum aestivum L.). Theor. Appl. Genet. 111:325336.

2. Bai, D., and Knott, D. R. 1992. Suppression of rust resistance in bread wheat (Triticum aestivum L.) by D-genome chromosomes. Genome 35:276-282.

3. Cai, X., Jones, S. S., and Murray, T. D. 1996. Characterization of an Agropyron elongatum chromosome conferring resistance to Cephalosporium stripe in common wheat. Genome 39:56-62.

4. Cenci, A., D'Ovidio, R., Tanzarella, O. A., Ceoloni, C., Pasquini, M., and Porceddu, E. 2003. Genetic analysis of the Aegilops longissima $3 \mathrm{~S}$ chromosome carrying the Pm13 resistance gene. Euphytica 130:177-183.

5. Chen, P. D., Tsujimoto, H., and Gill, B. S. 1994. Transfer of $P h^{I}$ genes promoting homoeologous pairing from Triticum speltoides to common wheat. Theor. Appl. Genet. 88:97-101.
6. Ciuffetti, L. M., Tuori, R. P., and Gaventa, J. M. 1997. A single gene encodes a selective toxin causal to the development of tan spot of wheat. Plant Cell 9:2135-2144.

7. De Wolf, E. D., Effertz, R. J., Ali, S., and Francl, L. J. 1998. Vistas of tan spot research. Can. J. Plant Pathol. 20:349-444.

8. Effertz, R. J., Meinhardt, S. W., Anderson, J. A., Jordahl, J. G., and Francl, L. J. 2002. Identification of a chlorosis-inducing toxin from Pyrenophora tritici-repentis and the chromosomal location of an insensitivity locus in wheat. Phytopathology 92:527-533.

9. Eyal, Z. 1999. The Septoria tritici and Stagonospora nodorum blotch diseases of wheat. Eur. J. Plant Pathol. 105:629-641.

10. Faris, J. D., and Friesen, T. L. 2005. Identification of quantitative trait loci for racenonspecific resistance to tan spot in wheat. Theor. Appl. Genet. 111:386-392.

11. Friebe, B., Jiang, J., Raupp, W. J., McIntosh, R. A., and Gill, B. S. 1996. Characterization of wheat-alien translocations conferring resistance to diseases and pests: current status. Euphytica 91:59-87.

12. Fried, P. M., and Meister, E. 1987. Inheritance of leaf and head resistance of winter wheat to Septoria nodorum in a diallel cross. Phytopathology 77:1371-1375.

13. Friesen, T. L., Ali, S., Kianian, S., Francl, L. J., and Rasmussen, J. B. 2003. Role of host sensitivity to Ptr ToxA in development of tan spot of wheat. Phytopathology 93:397-401.

14. Friesen, T. L., and Faris, J. D. 2004. Molecular mapping of resistance to Pyrenophora triticirepentis race 5 and sensitivity to $P \operatorname{tr}$ ToxB in wheat. Theor. Appl. Genet. 109:464-471.

15. Friesen, T. L., Meinhardt, S. W., and Faris, J. D. 2007. The Stagonospora nodorum-wheat pathosystem involves multiple proteinaceous host-selective toxins and corresponding host sensitivity genes that interact in an inverse gene-for-gene manner. Plant J. 51:681-692.

16. Friesen, T. L., Rasmussen, J. B., Kwon, C. Y., Ali, S., Francl, L. J., and Meinhardt, S. W. 2002. Reaction of Ptr ToxA-insensitive wheat mutants to Pyrenophora tritici-repentis race 1. Phytopathology 92:38-42.

17. Friesen, T. L., Stukenbrock, E. H., Liu, Z., Meinhardt, S., Ling, H., Faris, J. D., Rasmussen, J. B., Solomon, P. S., McDonald, B. A., and Oliver, R. P. 2006. Emergence of a new disease as a result of interspecific virulence gene transfer. Nat. Genet. 38:953-956.

18. Gamba, F. M., and Lamari, L. 1998. Mendelian inheritance of resistance to tan spot (Pyrenophora tritici-repentis) in selected genotypes of durum wheat (Triticum turgidum) Can. J. Plant Pathol. 20:408-414.

19. Gamba, F. M., Lamari, L., and Brule-Babel, A. 1998. Inheritance of race-specific necrotic and chlorotic reactions induced by Pyrenophora tritici-repentis in hexaploid wheats. Can. J. Plant Pathol. 20:401-407.

20. Jiang, J., Friebe, B., and Gill, B. S. 1994. Recent advances in alien gene transfer in wheat. Euphytica 73:199-212.

21. Jones, S. S., Murray, T. D., and Allan, R. E. 1995. Use of alien genes for the development of disease resistance in wheat. Annu. Rev. Phytopathol. 33:429-443.

22. Lamari, L., and Bernier, C. C. 1989. Evaluation of wheat lines and cultivars to tan spot (Pyrenophora tritici-repentis) based on lesion type. Can. J. Plant Pathol. 11:49-56.

23. Lamari, L., and Bernier, C. C. 1991. Genetics of $\tan$ necrosis and extensive chlorosis in tan spot of wheat caused by Pyrenophora triticirepentis. Phytopathology 81:1092-1095.

24. Lamari, L., McCallum, B. D., and DePauw, R. M. 2005. Forensic pathology of Canadian bread wheat: The case of tan spot. Phytopathology 95:144-152.

25. Liu, Z. H., Faris, J. D., Meinhardt, S. W., Ali, 
S., Rasmussen, J. B., and Friesen, T. L. 2004. Genetic and physical mapping of a gene conditioning sensitivity in wheat to a partially purified host-selective toxin produced by Stagonospora nodorum. Phytopathology 94:10561060.

26. Liu, Z. H., Friesen, T. L., Ling, H., Meinhardt, S. W., Oliver, R. P., Rasmussen, J. B., and Faris, J. D. 2006. The Tsn1-ToxA interaction in the wheat-Stagonospora nodorum pathosystem parallels that of the wheat-tan spot system. Genome 49:1265-1273.

27. Liu, Z. H., Friesen, T. L., Rasmussen, J. B., Ali, S., Meinhardt, S. W., and Faris, J. D. 2004. Quantitative trait loci analysis and mapping of seedling resistance to Stagonospora nodorum leaf blotch in wheat. Phytopathology 94:10611067.

28. Manning, V. A., and Ciuffetti L. M. 2005. Localization of Ptr ToxA produced by Pyrenophora tritici-repentis reveals protein import into wheat mesophyll cells. Plant Cell 17:3203-3212.

29. Martinez, J. P., Ottum, S. A., Ali, S., Francl, L. J., and Ciuffetti, L. M. 2001. Characterization of the ToxB gene from Pyrenophora triticirepentis. Mol. Plant-Microbe Interact. 14:675677.

30. Mathre, D. E., Johnston, R. H., and Martin, J.
M. 1985. Sources of resistance to Cephalosporium graminearum in Triticum and Agropyron species. Euphytica 34:419-424.

31. McIntosh, R. A., Wellings, C. R., and Park, R. F. 1995. Wheat rusts: an atlas of resistance genes. CSIRO Australia/Kluwer Academic Publishers, Dordrecht, The Netherlands.

32. Oliver, R. E., Cai, X., Xu, S. S., Chen, X., and Stack, R. W. 2005. Wheat-alien species derivatives: a novel source of resistance to Fusarium head blight in wheat. Crop Sci. 45:1353-1360.

33. Rees, R. G., and Platz, G. J. 1990. Sources of resistance to Pyrenophora tritici-repentis in bread wheats. Euphytica 45:59-69.

34. Rees, R. G., and Platz, G. J. 1992. Tan spot and its control-some Australian experiences. Pages 1-9 in: Advances in Tan Spot Research. Proc. 2nd Int. Tan Spot Workshop. N. D. Agric. Exp. Stn. L. J. Francl, J. M. Krupinsky, and M. P. McMullen, eds. Fargo, ND.

35. Riede, C. R., Francl, L. J., Anderson, J. A., Jordahl, J. G., and Meinhardt, S. W. 1996. Additional sources of resistance to tan spot of wheat. Crop Sci. 36:771-777.

36. Sharma, D., and Knott, D. R. 1966. The transfer of leaf rust resistance from Agropyron to Triticum by irradiation. Can. J. Genet. Cytol. 8:137-143.
37. Stover, R. W., Francl, L. J., and Jordahl, J. G. 1996. Tillage and fungicide management of foliar disease in a spring wheat monoculture. $\mathrm{J}$. Prod. Agric. 9:261-265.

38. Sykes, E. E., and Bernier, C. C. 1991. Qualitative inheritance of tan spot resistance in hexaploid, tetraploid, and diploid wheat. Can. J. Plant Pathol. 13:38-44.

39. Wang, R. R.-C., and Zhang, X.-Y. 1996. Characterization of the translocated chromosome using fluorescence in situ hybridization and random amplified polymorphic DNA on two Triticum aestivum-Thinopyrum intermedium translocation lines resistant to wheat streak mosaic or barley yellow dwarf virus. Chromatogr. Res. 4:583-587.

40. Wicki, W., Winzeler, M., Schmid, J. E., Stamp, P., and Messmer, M. 1999. Inheritance of resistance to leaf and glume blotch caused by Septoria nodorum Berk. in winter wheat. Theor Appl. Genet. 99:1265-1272.

41. Xu, S. S., Friesen, T. L., and Cai, X. 2004 Sources and genetic control of resistance to Stagonospora nodorum blotch in wheat. Recent Res. Dev. Genet. Breed. 1:449-469.

42. Xu, S. S., Friesen, T. L., and Mujeeb-Kazi, A. 2004. Seedling resistance to tan spot and Stagonospora nodorum blotch in synthetic hexaploid wheats. Crop Sci. 44:2238-2245. 